\title{
CONTRIBUTION TO THE FLORA OF MÁLAGA
}

\author{
B. E. SMYthiES*
}

\section{SUMMARY}

A list is given of plants collected or sighted by the author in Málaga province or nearby.

\section{RESUMEN}

Se da una lista de plantas recolectadas o vistas por el autor en la provincia de Málaga o localidades limítrofes.

\section{PTERIDOPHYTA}

EQUISETACEAE

\section{Equisetum ramossisimum Desf.}

UF03 Sierra Bermeja, 17-V-73, Smythies (Herb. LTR 802).

\section{SINOPTERIDACEAE}

Cheilanthes catanensis (Cosent.) H.P. Fuchs

UF14 Benahavís (v.v. 28-III-1973).

Cheilanthes fragans (L. fil.) Swartz

UF03 Sierra Bermeja (v.v. 3-V-1972).

UF14 Benahavís (v.v. 28-III-1973).

(*) Kew Gardens, Surrey (England) / San Diego, Estepona, Málaga (Spain). 
Cheilanthes marantae (L.) Domin.

UF03 Sierra Bermeja (v.v. 28-IV-1973).

UF04 Sierra Bermeja (v.v. 3-II-1973).

UF14 Benahavís (v.v. 28-III-1973).

\section{ADIANTACEAE}

Adiantum capillus-veneris $\mathrm{L}$.

UF03 Sierra Bermeja (v.v. 6-V-1968).

\section{GYNNOGRAMNACEAE}

Anogramma leptophylla (L.) Link.

UF03 Sierra Bermeja (v.v. 3-V-1972).

\section{DAVALLIACEAE}

Davallia canariensis (L.) Sm.

UF03 Sierra Bermeja (v.v. 3-V-1972).

\section{ASPLENIACEAE}

Asplenium adiantum-nigrum $\mathrm{L}$.

TF93 way to Majada Madrid (v.v. 14-II-1974).

UF03 Río Padrón (v.v. 19-II-1973).

Asplenium cuneifolium Viv.

UF04 Sierra Bermeja (v.v. 3-V-1972).

Asplenium onopteris L.

UF04 Sierra Bermeja (v.v. 3-V-1972).

Asplenium petrarchae (Guérin.) DC.

TF96 Montejaque (v.v. 21-V-1975). 
Asplenium trichomanes $\mathrm{L}$.

UF03 Sierra Bermeja (v.v. 3-V-1972).

\section{ATHYRIACEAE}

Athyrium filix-femina (L.) Roth

UF03 Sierra Bermeja (v.v. 3-V-1972).

POLYPODIACEAE

Polypodium australe Fée

UF03 Sierra Bermeja (v.v. 3-V-1972).

CONIFEROPSIDA

CONIFERALES

PINACEAE

Pinus halepensis Miller

TF94 Sierra Espartina (v.v. 28-V-1975).

Pinus pinea $\mathrm{L}$.

UF15 Coto Ragete (v.v. 28-V-1975).

\section{CUPRESSACEAE}

Juniperus sabina $L$.

UF16 Sierra de las Nieves (v.v. 29-V-1975).

UF26 Torrecilla (v.v. 6-VI-1974).

TAXOPSIDA

TAXALES

TAXACEAE

Taxus baccata $\mathrm{L}$.

UF26 Torrecilla (v.v. 6-VI-1974). 


\section{GNETOPSIDA \\ GNETALES \\ EPHEDRACEAE}

\section{Ephedra fragilis Desf.}

UF34 Sierra Blanca (v.v. 13-V-1973).

UF55 Sierra de Mijas (v.v. 23-IV-1975).

SPERMATOPHYTA

ANGIOSPERMAE

DICOTYLEDONES

SALICALES

SALICACEAE

Salix pedicellata Desf.

UF03 Sierra Bermeja, 12-V-1971, Smythies (Herb. LTR 566).

FAGALES

FAGACEAE

Quercus faginea Lam.

UF03 Río Padrón (v.v. 19-II-1973).

UF16 Sierra de las Nieves (v.v. 29-V-1975) [var. alpestris Boiss.]

VF17 Sierra Tejeda, 25-V-1974, Smythies (Herb. LTR 938).

Quercus rotundifolia $\backslash$ Lam.

UF07 Setenil (v.v. 10-V-1972).

URTICALES

ULMACEAE

Celtis australis $\mathrm{L}$.

UF14 Benahavís (v.v. 21-V-1974).

VF93 Sierra Crestellina (v.v. 12-V-1975). 
Parietaria lusitanica L.

TF93 Sierra Crestellina (v.v. 12-V-1975).

SANTALALES

SANTALACEAE

Thesium divaricatum Jan. ex Mert. \& Koch

UF04 Sierra Bermeja (v.v. 3-V-1972).

\section{LORANTHACEAE}

Viscum cruciatum Sieber ex Boiss.

UF26 Sierra de las Nieves (v.v. 3-IV-1972).

\section{RAFFLESIACEAE (CYTINACEAE)}

Cytinus ruber (Fourr.) Komarov

UF03 Sierra Bermeja (v.v. 28-IV-1973).

UF14 Benahavís (v.v. 28-III-1973).

UF37 Sierra de Alcaparaín (v.v. 23-IV-1970).

\section{POLYGONALES}

POLYGONACEAE

Rumex induratus Boiss. \& Reuter

UF03 Sierra Bermeja (v.v. 7-IV-1972).

\section{CENTROSPERMAE}

CARYOPHYLLACEAE

Arenaria aggregata (L.) Loisel

UF26 Sierra de las Nieves (v.v. 28-V-1974). 
Arenaria capillipes Boiss.

UF04 Sierra Bermeja, 28-V-1970, Smythies (Herb. LTR 434).

Arenaria modesta Dufour

UF34 Sierra Blanca (v.v. 13-V-1973).

Arenaria montana $\mathrm{L}$.

TF93 Sierra Crestellina (v.v. 26-IV-1968).

UF03 Sierra Bermeja, 9-V-1969, Smythies (Herb. LTR 340).

Arenaria pomelii Munby

UF04 Sierra Bermeja, 7-IV-1972, Smythies (Herb. LTR 672).

UF04 Sierra Bermeja, 28-IV-1973, Smythies (Herb. LTR 779).

Arenaria retusa Boiss.

UF03 Sierra Bermeja, 7-IV-1972, Smythies (Herb. LTR 671).

UF14 Benahavís (v.v. 28-III-1973).

Minuartia geniculata (Poiret) Thell.

VF26 Cueva de Nerja (v.v. 13-IV-1967).

Stellaria neglecta Weihe

UF03 Río Padrón (v.v. 19-II-1973).

UF14 Benahavís (v.v. 22-II-1973).

Cerastium boissieri Gren.

UF98 Ventas de Zafarraya (Granada) (v.v. 13-V-1970).

VF17 above Canillas de Albaida, 6-V-1969, Smythies (Herb. LTR 329).

Corrigiola telephilifolia Pourret

UF04 Sierra Bermeja (v.v. 16-IV-1975).

UF14 Benahavís (v.v. 10-VI-1973).

UF43 Artola (v.v. 16-II-1966).

\section{Scleranthus annuus $\mathrm{L}$.}

UF16 Sierra de las Nieves (v.v. 29-V-1975). 
Herniaria hirsuta L.

UF55 Sierra de Mijas (v.v. 23-IV-1975).

Herniaria scabrida Boiss.

UF04 Sierra Bermeja, 3-V-1972, Smythies (Herb. LTR 673).

UF35 Carretera 337, 11-VI-1975, Smythies (Herb. LTR 1117).

\section{Silene stricta $\mathrm{L}$.}

UG40 Laguna Salada, 19-V-1968, Smythies (Herb. E 262).

Silene colorata Poiret

UF06 Carretera 339 (v.v. 4-III-1966).

UF07 Carretera 339 (v.v. 4-III-1966).

Silene divaricata Clemente

VF17 above Canillas de Albaida, 6-V-1969, Smythies (Herb. LTR 332).

Silene littorea Brot.

UF55 Sierra de Mijas, 26-V-1974, Smythies (Herb. LTR 949).

Silene psammitis Link ex Sprengel

UF14 Benahavís, 25-IV-1966, Smythies (Herb. E 81).

UF35 Carretera 337, 11-VI-1975, Smythies (Herb. LTR 1116).

VF17 above Canillas de Albaida (v.v. 6-V-1969).

Silene pseudovelutina Rothm.

TF93 Sierra Crestellina (v.v. 12-V-1975).

UF06 Montejaque (v.v. 6-V-1967).

UF98 Ventas de Zafarraya (Granada), 13-V-1970, Smythies (Herb. LTR 410.)

Petrorhagia velutina (Guss.) P. W. Ball. \& Heywood

UF14 Benahavís (v.v. 28-III-1973).

Dianthus hispanicus Asso

VF18 south of Jatar (Granada), 14-V-1970, Smythies (Herb. LTR 415). 
RANALES

RANUNCULACEAE

Delphinium obcordatum DC.

UF14 Benahavís (v.v. 10-VI-1973).

Delphinium staphisagria $\mathrm{L}$.

UF02 Carretera 340 (v.v. 20-IV-1967).

Anemone palmata L.

UF37 Sierra de Alcaparaín (v.v. 14-IV-1967).

Ranunculus gramineus $\mathrm{L}$.

UF34 Sierra Blanca (v.v. 15-IV-1967).

UF55 Sierra de Mijas (v.v. 25-V-1971).

Ranunculus paludosus Poiret

UF04 Sierra Bermeja (v.v. 12-V-1971).

\section{PAEONIACEAE}

Paeonia broteroi Boiss. \& Reuter

UF06 Montejaque (v.v. 6-V-1967).

UF15 Carretera 339 (v.v. 4-V-1967).

Paeonia coriacea Boiss.

UF16 Sierra de las Nieves (v.v. 6-VI-1974).

\section{BERBERIDACEAE}

Berberis hispanica Boiss. \& Reuter

UF26 Torrecilla (v.v. 6-VI-1974).

VF08 Sierra Tejeda (v.v. 25-V-1974). 
RHOEDALES

PAPAVERACEAE

\section{Papaver hybridum $L$.}

TF98 El Castor (v.v. 27-V-1975).

Fumaria macrosepala Boiss.

TF93 Sierra Crestellina (v.v. 12-V-1975).

Rupicapnos africana (Lam.) Pomel

TF93 Casares (v.v. 31-V-1974).

UF48 Camino del Rey (v.v. 10-V-1974).

\section{CAPPARIDACEAE}

Cleome violacea $\mathrm{L}$.

UF03 Sierra Bermeja (v.v. 3-VI-1975).

UF14 Carretera 339, 5-IV-1966, Smythies (Herb. E. 65).

\section{CRUCIFERAE}

Sisymbrium arundanum Boiss.

UF48 Presa de Gobantes, 10-V-1974, Smythies (Herb. LTR 908).

Erysimum linifolium (Pers.) Gay

VF17 Cortijo del Daire (v.v. 17-V-1972).

Hesperis laciniata All.

TF93 Sierra Crestellina (v.v. 12-V-1975).

UF98 Ventas de Zafarraya (Granada), 25-III-1966, Smythies (Herb. E 50).

Matthiola fruticulosa (L.) Maire

UF55 Sierra de Mijas, 25-V-1971, Smythies (Herb. LTR 576). 
Arabis verna (L.) R. Br.

TF93 Sierra Crestellina (v.v. 26-IV-1968).

UF14 Benahavís (v.v. 22-III-1968).

UF35 Sierra Blanca (v.v. 15-IV-1967).

UF69 El Torcal (v.v. 28-III-1966).

Alyssum minus (L.) Rothm.

UF03 Sierra Bermeja (v.v. 6-V-1968).

\section{Alyssum montanum L.}

VF17 above Canillas de Albaida, 4-V-1969, Smythies (Herb. LTR 337).

\section{Alyssum serpyllifolium Desf.}

UF15 Carretera 339, 25-XII-1966, Smythies (Herb. E. 141).

Ptilotrichum spinosum (L.) Boiss.

UF26 Torrecilla (v.v. 6-VI-1974).

Lobularia maritima (L.) Desv.

UF15 Carretera 339 (v.v. 25-XII-1966).

Draba hispanica Boiss.

UF16 Sierra de las Nieves (v.v. 7-V-1967).

VF08 Sierra Tejeda (v.v. 25-V-1974).

Erophila verna (L.) Chevall.

UF26 Sierra de las Nieves (v.v. 3-IV-1972).

Hornungia petraea (L.) Reichenb.

UF16 Sierra de las Nieves (v.v. 29-V-1975).

Ionopsidium prolongoi (Boiss.) Batt.

TF85 Peñón de Berrueco, 5-V-1971, Smythies (Herb. LTR 558).

UF16 Sierra de las Nieves, 6-VI-1974, Smythies (Herb. LTR. 959).

UF16 Sierra de las Nieves, 29-V-1975, Smythies (Herb. LTR 1082). 
Aethionema saxatile (L.) R. Br.

UF26 Sierra de las Nieves (v.v. 7-V-1967).

Iberis crenata Lam.

UF06 Ronda (v.v. 6-V-1967).

UF14 Benahavís, 28-III-1973, Smythies (Herb. LTR 754).

UF48 Sierra de la Pizarra (v.v. 14-IV-1967).

Iberis fontqueri Pau

UF03 Sierra Bermeja, 17-V-1973, Smythies (Herb. LTR 803).

Iberis linifolia Loefl.

UF35 Sierra Blanca (v.v. 13-V-1973).

UF47 Sierra de Aguas (v.v. 18-V-1970).

UF65 Sierra Llana (v.v. 24-V-1971).

Iberis pruitii Tineo

UF26 Sierra de las Nieves (v.v. 7-V-1967).

UF37 Sierra de Alcaparaín (v.v. 23-IV-1970).

Iberis saxatilis $\mathrm{L}$.

VF17 above Canillas de Albaida, 6-V-1969, Smythies (Herb. LTR 333).

Biscutella frutescens Cosson

UF35 Sierra Blanca (v.v. 15-IV-1967).

UF98 Ventas de Zafarraya (Granada) (v.v. 25-III-1966).

VF17 above Canillas de Albaida (v.v. 6-V-1969).

Biscutella Iusitanica Jordan

TF96 Cueva de la Pileta, 21-V-1974, Smythies (Herb. LTR 912).

UF16 Sierra de las Nieves, 6-VI-1974, Smythies (Herb. LTR 964).

Biscutella sempervirens $\mathrm{L}$.

UF04 Sierra Bermeja, 3-V-1972, Smythies (Herb. LTR 675).

UF04 Sierra Bermeja, 20-V-1974, Smythies (Herb. LTR 910).

UF05 Sierra Bermeja (v.v. 7-IV-1972).

Biscutella valentina (L.) Heywood

UF16 Sierra de las Nieves, 6-VI-1974, Smythies (Herb. LTR 960). 


\section{Lepidium hirtum $\mathrm{Sm}$.}

subsp calycotrichum (G. Kunze) Thell.

UF16 Sierra de las Nieves, 29-V-1975, Smythies (Herb. LTR 1081).

Moricandia moricandioides (Boiss.) Heywood

UF06 Ronda (v.v. 3-IV-1969).

Vella spinosa Boiss.

VF08 Sierra Tejeda (v:v. 25-V-1974).

Crambe filiformis Jacq.

UF06 Ronda (v.v. 6-V-1967).

\section{RESEDACEAE}

Reseda alba L.

UF06 Ronda (v.v. 10-V-1972).

Reseda phyteuma L.

UF03 Sierra Bermeja (v.v. 6-V-1968).

Reseda suffruticosa Loefl.

UF35 Sierra Blanca (v.v. 29-III-1967).

UF37 Sierra de Alcaparaín (v.v. 14-IV-1967).

UF55 Sierra de Mijas (v.v. 23-IV-1975).

Reseda undata L.

$=R$. gayana Boiss.

TF96 Montejaque, 21-V-1975, Smythies (Herb. LTR 1077).

\section{ROSALES}

CRASSULACEAE

Umbilicus horizontalis (Guss.) DC.

UF14 Benahavís (v.v. 21-V-1974). 
Mucizonia hispida (Lam.) A. Berger

UF06 Ronda (v.v. 6-V-1967).

Sedum tenuifolium (S.bth \& Sm.) Strobl

UF03 Sierra Bermeja, 11-VI-1974, Smythies (Herb. LTR 973).

UF06 Montejaque (v.v. 21-V-1975).

\section{SAXIFRAGACEAE}

\section{Saxifraga biternata Boiss.}

UF69 El Torcal, 19-V-1968, Smythies (Herb. E 264).

UF89 Sierra de Camorolos (v.v. 24-V-1974).

\section{Saxifraga boissieri Engler}

TF85 Peñón de Berruecos, 5-V-1971, Smythies (Herb. LTR 559).

TF96 Cueva de la Pileta (v.v. 21-V-1974).

UF06 Montejaque (v.v. 21-V-1974).

Saxifraga dichotoma Sternb.

subsp. dichotoma

UF16 Sierra de las Nieves, 29-V-1975, Smythies (Herb. LTR 1086).

UF26 Sierra de las Nieves (v.v.. 28-V-1974).

subsp. albarracinensis (Pau) D. A. Webb.

UF16 Sierra de las Nieves, 29-V-1975, Smythies (Herb. LTR 1086).

UF26 Sierra de las Nieves (v.v. 28-V-1974).

Saxifraga erioblasta Boiss.

VF08 Sierra Tejeda, 25-V-1974, Smythies (Herb. LTR 935).

Saxifraga gemmulosa Boiss.

UF04 Sierra Bermeja (v.v. 28-V-1970).

UF15 Carretera 339 (v.v. 4-V-1967).

Saxifraga globulifera Desf.

TF93 Sierra Crestellina (v.v. 12-V-1975).

UF06 Montejaque (v.v. 21-V-1974). 
UF14 Benahavís (v.v. 22-III-1968).

UF15 Carretera 339 (v.v. 21-V-1974).

UF16 Carretera 339 (v.v. 21-V-1974).

UF26 Sierra de las Nieves (v.v. 28-V-1974).

UF34 Sierra Blanca (v.v. 13-V-1973).

UF37 Sierra de Alcaparaín (v.v. 18-V-1970).

UF55 Sierra de Mijas (v.v. 25-V-1971).

UF65 Sierra Llana (v.v. 26-V-1974).

\section{Saxifraga granulata L.}

UF03 Benahavís (v.v. 3-V-1972).

UF06 Montejaque (v.v. 21-V-1974).

UF14 Carretera 339, 19-II-1966, Smythies (Herb. E 24).

UF26 Torrecilla (v.v. 6-VI-1974).

VF08 Sierra Tejeda (v.v. 25-V-1974).

Saxifraga haenseleri Boiss. \& Reuter

VF08 Sierra Tejeda (v.v. 25-V-1974).

Saxifraga reuterana Boiss.

UF49 Sierra de Abdalajís, 24-V-1974, Smythies (Herb. LTR 931).

UF69 El Torcal, 23-V-1974, Smythies (Herb. LTR 925).

UF79 Sierra de las Cabras (v.v. 24-V-1974).

UF89 Sierra de Camorolos (v.v. 24-V-1974).

Saxifraga tridactylites $\mathrm{L}$.

UF06 Ronda (v.v. 2-IV-1974).

UF16 Sierra de las Nieves (v.v. 29-V-1975).

VF08 Sierra Tejeda (v.v. 25-V-1974).

\section{ROSACEAE}

Rosa glauca Pourret

VF08 Sierra Tejeda (v.v. 25-V-1974).

Sanguisorba minor Scop.

UF04 Sierra Bermeja (v.v. 4-IV-1967). 
Crataegus monogyna Jacq.

UF26 Sierra de las Nieves (v.v. 3-IV-1972).

Prunus prostrata Labill.

UF26 Sierra de las Nieves (v.v. 7-V-1967).

VF08 Sierra Tejeda (v.v. 25-V-1974).

\section{LEGUMINOSAE}

Cytisus baeticus (Webb.) Steudel

UF04 Sierra Bermeja (v.v. 7-IV-1972).

UF06 Ronda (v.v. 11-IV-1974).

UF14 Benahavís (v.v. 27-XI-1969).

Cytisus grandiflorus DC.

UF08 Sierra Tejeda (v.v. 25-V-1974).

UF15 Coto Ragete (v.v. 6-VI-1974).

Cytisus striatus (Hill.) Rothm.

UF04 Sierra Bermeja (v.v. 16-IV-1975).

Teline monspessulana (L.) C. Koch

TF93 way to Majada Madrid (v.v. 3-XII-1969).

Genista haenseleri Boiss.

UF35 Carretera 337, 11-VI-1975, Smythies (Herb. LTR 1115).

Genista hirsuta Vahl

UF03 Sierra Bermeja (v.v. 12-V-1971).

UF04 Sierra Bermeja (v.v. 12-V-1971).

UF14 Carretera 339 (v.v. 19-II-1966).

Genista lanuginosa Spach

UF03 Sierra Bermeja (v.v. 12-V-1971).

UF04 Sierra Bermeja (v.v. 12-V-1971). 
Retama sphaerocarpa Boiss.

TF96 Benahavís (v.v. 25-IV-1966).

UF98 Carretera 335 (v.v. 13-V-1970).

VF17 Carretera 335 (v.v. 13-V-1970).

Spartium junceum L.

VF08 north of Alcaucín (v.v. 8-VI-1975).

Erinacea anthyllis Link

UF04 Sierra Bermeja (v.v. 28-V-1970).

UF26 Sierra de las Nieves (v.v. 7-V-1967).

Ulex parviflorus Pourret

UF03 Sierra Bermeja (v.v. 12-V-1971).

UF04 Sierra Bermeja (v.v. 12-V-1971).

UF15 Carretera 339 (v.v. 25-XII-1966). [subsp. jussiaei (Webb.) D. A. Webb].

Adenocarpus decorticans Boiss.

VF18 c. Puerto de Cómpeta (v.v. 14-V-1970).

Adenocarpus telonensis (Loisel.) DC.

UF14 Carretera 339 (v.v. 19-II-1966).

Lotononis lupinifolia (Boiss.) Bentham

UF24 Istán road (v.v. 3-V-1975).

VF08 north of Alcaucín (v.v. 8-VI-1975).

\section{Lupinus albus $\mathrm{L}$.}

TF95 Atajate (v.v. 11-IV-1974).

Lupinus hispanicus Boiss. et Reuter

TF94 Genalguacil, 10-V-1972, Smythies (Herb. LTR 697).

Argyrolobium zanonii (Turra) P.W. Ball

TF94 Sierra Espartina (v.v. 5-V-1975).

UF14 Benahavís (v.v. 13-IV-1973).

UF55 Sierra de Mijas (v.v. 23-IV-1975).

VF17 Above Canillas de Albaida, 18-V-1972, Smythies (Herb. LTR 704). 
Colutea atlantica Browicz

UF14 Benahavís, 27-I-1966, Smythies (Herb. E 12).

Astragalus epiglottis L.

UF16 Sierra de las Nieves (v.v. 29-V-1975).

Astragalus granatensis Lam.

UF26 Torrecilla (v.v. 6-VI-1974).

VF08 Sierra Tejeda (v.v. 25-V-1974).

Psoralea bituminosa L.

VF17 way to Cortijo del Daire (v.v. 17-V-1972).

Vicia disperma DC.

UF03 Sierra Bermeja (v.v. 3-V-1972).

Vicia pubescens (DC.) Link

UF03 Sierra Bermeja (v.v. 28-IV-1973).

Vicia sativa $L$.

UF03 Sierra Bermeja (v.v. 28-IV-1973).

Vicia tenuissima (Bieb.) Schinz \& Thell.

UF16 Sierra de las Nieves (v.v. 29-V-1975).

Vicia vicioides (Desf.) Coutinho

TF85 Peñón de Berrueco, 22-V-1974, Smythies (Herb. LTR 921).

UF48 Sierra de la Pizarra, 28-III-1967, Smythies (Herb. E 167).

\section{Lathyrus angulatus $\mathrm{L}$.}

UF14 Benahavís (v.v. 28-III-1973).

\section{Lathyrus cicera $\mathrm{L}$.}

UF14 Benahavís (v.v. 28-III-1973).

\section{Lathyrus setifolius $\mathrm{L}$.}

UF69 El Torcal (v.v. 23-V-1974). 
Ononis aragonensis Asso

VF08 Sierra Tejeda (v.v. 25-V-1974).

Ononis natrix $\mathrm{L}$.

VF17 above Canillas de Albaida (v.v. 6-V-1969).

Ononis pendula Desf.

UF14 Carretera 339, 5-IV-196, Smythies (Herb. E 66).

Ononis sicula Guss.

TF94 Sierra Espartina (v.v. 5-V-1975).

Ononis speciosa Lag.

UF14 Benahavís (v.v. 3-V-1970).

UF35 Sierra Blanca (v.v. 15-IV-1967).

UF69 Carretera 331 (v.v. 29-III-1966).

Ononis viscosa $\mathrm{L}$.

UF14 Benahavís (v.v. 28-IV-1973).

Trigonella ovalis Boiss.

UF15 Igualeja (v.v. 28-V-1975).

Dorycnium pentaphyllum Scop.

VF08 north of Alcaucín (v.v. 8-VI-1975).

Doryenium rectum (L.) Ser.

VF08 north of Alcaucín (v.v. 8-VI-1975).

Anthyllis cytisoides $\mathrm{L}$.

UF14 Carretera 339 (v.v. 19-II-1966).

UF35 Sierra Blanca (v.v. 15-IV-1967).

Anthyllis polycephala Desf.

UF03 Sierra Bermeja, 12-V-1971, Smythies (Herb. LTR 567).

UF14 Benahavís (v.v. 28-III-1973).

UF37 Sierra de Alcaparaín (v.v. 14-IV-1967). 


\section{Anthyllis tejedensis Boiss.}

VF08 Sierra Tejeda, 25-V-1974, Smythies (Herb. LTR 937).

VF17 Above Canillas de Albaida, 6-V-1969, Smythies (Herb. LTR 327).

VF17 Above Canillas de Albaida, 18-V-1972, Smythies (Herb. LTR 707).

Anthyllis vulneraria $\mathrm{L}$.

UF14 Benahavís (v.v. 25-IV-1966).

UF37 Carratraca, 6-III-1966, Smythies (Herb. E 39).

Ornithopus compressus $\mathrm{L}$.

UF14 Benahavís (v.v. 28-III-1973).

Coronilla juncea L.

UF14 Sierra Bermeja (v.v. 12-V-1971).

Coronilla valentina $\mathrm{L}$.

UF14 Carretera 339 (v.v. 19-II-1966).

Hippocrepis ciliata Willd.

VF17 above Canillas de Albaida, 6-V-1969, Smythies (Herb. LTR 335).

Hedysarum glomeratum F.G. Dietr.

TF94 Sierra Espartina (v.v. 5-V-1975).

Hedysarum humile L.

UF69 Torcal, 28-III-1966, Smythies (Herb. E 62).

Onobrychis argentea Boiss.

UF69 El Torcal (v.v. 28-III-1966).

\section{GERANIALES}

GERANIACEAE

\section{Geranium lucidum L.}

TF93 Sierra Crestellina (v.v. 26-IV-1968).

UF69 EL Torcal (v.v. 9-V-1967). 
Geranium malviflorum Boiss. \& Reuter

TF95 Jimera de Líbar (v.v. 10-V-1972).

UF06 Ronda (v.v. 11-IV-1974).

Erodium cheilanthifolium Boiss.

UF26 Sierra de las Nieves, 6-VI-1974, Smythies (Herb. LTR 965).

VF08 Sierra Tejeda (v.v. 25-V-1974). Smythies.

Erodium guttatum (Desf.) Willd.

UF26 Yunquera (v.v. 18-V-1976).

Erodium laciniatum (Cav.) Willd.

UF35 Sierra Blanca, 29-III-1967 (Gerb. E 164).

Erodium moschatum (L.) L'Hér.

UF03 Río Padrón (v.v. 19-II-1973).

\section{LINACEAE}

\section{Linum narbonense $L$.}

UF03 Sierra Bermeja, 4-IV-1967, Smythies (Herb. E 171).

UF47 Sierra de Aguas (v.v. 18-V-1970).

Linum setaceum Brot.

UF65 Sierra Llana, 26-V-1974, Smythies (Herb. LTR 942).

\section{Linum suffruticosum $\mathrm{L}$.}

UF14 Carretera 339, 5-IV-1966, Smythies (Herb. E 64).

UF47 Sierra de Aguas (v.v. 18-V-1970).

UF65 Sierra Llana (v.v. 24-V-1971).

\section{EUPHORBIACEAE}

Euphorbia flavicoma DC.

UF04 Sierra Bermeja, 12-V-1971, Smythies (Herb. LTR 562). 


\section{Euphorbia nicaeensis All.}

VF08 Sierra Tejeda, 25-V-1974, Smythies (Herb. LTR 940).

Euphorbia squamigera Loisel.

UF03 Sierra Bermeja, 12-V-1971, Smythies (Herb. LTR 565).

\section{RUTALES}

POLYGALACEAE

Polygala rupestris Pourret

UF14 Benahavís (v.v. 13-IV-1973).

UF48 El Chorro (v.v. 28-III-1967).

SAPINDALES

CORIARIACEAE

Coriaria myrtifolia L.

UF03 Sierra Bermeja (v.v. 12-V-1971).

UF04 Carretera de Istán (v.v. 28-IV-1968).

\section{ANACARDIACEAE}

\section{Rhus coriaria L.}

TF94 Carretera de Jubrique (v.v. 10-V-1972).

Pistacia terebinthus L.

TF93 Sierra Crestellina (v.v. 12-V-1965).

UF14 Benahavís (v.v. 22-III-1968).

UF35 Sierra Blanca (v.v. 15-IV-1967).

\section{ACERACEAE}

Acer granatense Boiss.

VF08 Sierra Tejeda, 25-V-1974, Smythies (Herb. LTR 939). 
Acer monspessulanum L.

UF89 Sierra de Camorolos, 24-V-1974, Smythies (Herb. LTR 928).

\author{
RHAMNALES \\ RHAMNACEAE
}

Rhamnus alaternus L.

UF04 Sierra Bermeja (v.v. 6-V-1968) [var. myrtifolius Willk.]

UF69 El Torcal, 19-V-1968, Smythies (Herb. E 263).

VF17 above Canillas de Albaida (v.v. 6-V-1969).

Rhamnus lycioides L.

subsp. oleoides (L.) Jahandiez et Maire

UF34 Sierra Blanca (v.v. 13-V-1973).

MALVALES

MALVACEAE

Malva hispanica L.

UF02 Carretera 340 (v.v. 5-IV-1966).

Lavatera cretica L.

UF02 Carretera 340 (v.v. 28-IV-1966).

Lavatera maritima Gouan

UF48 Camino del Rey (v.v. 10-V-1974).

UF55 Sierra de Mijas (v.v. 24-V-1971).

THYMELEALES

THYMELAEACEAE

Daphne laureola L.

UF26 Torrecilla (v.v. 6-VI-1974). 
Thymelaea nitida (Vahl.) Endl.

UF03 Sierra Bermeja (v.v. 20-V-1974).

Thymelaea tartonraira (L.) All.

VF17 above Canillas de Albaida, 6-V-1969, Smythies (Herb. LTR 338).

\section{VIOLALES \\ VIOLACEAE}

Viola demetria Prolongo ex Boiss.

UF17 Puerto del Viento, 4-III-1966, Smythies (Herb. E. 37).

UF26 Torrecilla, 6-VI-1974, Smythies (Herb. LTR 958).

UF69 El Torcal (v.v. 19-V-1968).

UF79 Sierra de las Cabras (v.v. 24-V-1974).

\section{CISTACEAE}

Cistus albidus $\mathrm{L}$.

UF35 Sierra Blanca (v.v. 15-IV-1967).

UF48 Sierra de la Pizarra (v.v. 14-IV-1967).

Cistus clusii Dunal

VF08 Sierra Tejeda (v.v. 25-V-1974).

VF17 above Canillas de Albaida (v.v. 17-V-1972).

VF18 south of Jatar (Granada) (v.v. 14-V-1970).

Cistus laurifolius $\mathrm{L}$.

UF15 Coto Ragete (v.v. 6-VI-1974).

Cistus populifolius $\mathrm{L}$.

UF03 Sierra Bermeja (v.v. 9-V-1969).

UF04 Sierra Bermeja (v.v. 9-V-1969).

Halimium atriplicifolium (Lam.) Spach

UF04 Sierra Bermeja (v.v. 28-V-1970).

UF14 Benahavís (v.v. 3-V-1970). 
UF15 Carretera 339 (v.v. 19-II-1966).

UF35 Sierra Blanca (v.v. 15-IV-1967).

Halimium halimifolium (L.) Willk.

UF14 Benahavís (v.v. 12-I-1968).

Tuberaria bupleurifolia (Lam.) Willk.

UF03 Sierra Bermeja, 28-IV-1973, Smythies (Herb. LTR 778).

Helianthemum appeninum (L.) Miller

UF26 Sierra de las Nieves (v.v. 7-V-1967).

UF55 Sierra de Mijas (v.v. 25-V-1971).

Helianthemum cinereum (Cav.) Pers.

UF26 Sierra de las Nieves, 28-V-1974, Smythies (Herb. LTR 951).

UF48 Sierra de la Pizarra (v.v. 28-III-1967).

UF55 Sierra de Mijas (v.v. 23-IV-1975).

VF17 above Canillas de Albaida (v.v. 6-V-1969).

Helianthemum croceum (Desf.) Pers.

UF55 Sierra de Mijas (v.v. 26-V-1974).

Helianthemum lavandulifolium Miller

UF02 Carretera 340 (v.v. 28-IV-1966).

Helianthemum marifolium (L.) Miller

UF24 La Concha (v.v. 24-II-1973).

Fumana thymifolia (L.) Spach ex Webb

UF24 Istán road (28-IV-1968).

MYRTALES

LYTHRACEAE

Lythrum junceum Banks et Solander

UF02 Carretera 340 (v.v. 28-IV-1966). 


\section{THELIGONACEAE}

Theligonum cynocrambe $\mathrm{L}$.

UF14 Benahavís (v.v. 22-II-1973).

\section{UMBELLIFLORAE \\ UMBELLIFERAE}

Eryngium aquifolium Cav.

UF03 Sierra Bermeja, 2-VI-1975, Smythies (Herb. LTR 1091).

Eryngium dilatatum Lam.

UF03 Sierra Bermeja, 11-VI-1974, Smythies (Herb. LTR 967).

Lagoecia cuminoides $\mathrm{L}$.

UF06 Montejaque (v.v. 21-V-1975).

Scandix australis $\mathrm{L}$.

UF06 Montejaque (v.v. 21-V-1974).

Scandix pecten-veneris $\mathrm{L}$.

UF14 Benahavís (v.v. 28-IV-1973).

Smyrnium olusatrum $\mathrm{L}$.

UF14 Benahavís (v.v. 3-V-1970).

Bunium alpinum Waldst. \& Kit.

UF16 Sierra de las Nieves, 6-VI-1974, Smythies (Herb. LTR 961).

Foeniculum vulgare Miller

UF14 Benahavís (v.v. 27-XI-1969).

Kundmannia sicula (L.) DC.

TF93 Casares road (v.v. 7-V-1970).

UF05 Alpandeire (v.v. 30-V-1975). 
Magydaris panacifolia (Vahl) Lange

UF37 Sierra de Alcaparaín (v.v. 18-V-1970).

UF89 Sierra de Camorolos, 24-V-1974, Smythies (Herb. LTR 929).

Bupleurum gerardi All.

UF15 Coto Ragete, 28-V-1975, Smythies (Herb. LTR 970).

\section{Bupleurum rigidum $\mathrm{L}$.}

UF03 Sierra Bermeja, 11-VI-1974, Smythies (Herb. LTR 970).

Bupleurum spinosum Gouan

UF26 Sierra de las Nieves (v.v. 26-III-1972).

Ferula communis L.

UF48 Camino del Rey (v.v. 10-V-1974).

UF55 Sierra de Mijas (v.v. 25-V-1971).

Thapsia garganica $\mathrm{L}$.

TF93 Sierra Crestellina, 7-V-1970, Smythies (Herb. LTR 405).

Elaeoselinum asclepium (L.) Bertol.

UF03 Sierra Bermeja, 11-VI-1974, Smythies (Herb. LTR 972).

Orlaya kochii Heywood

UF14 Benahavís (v.v. 28-IV-1973).

UF24 Carretera de Istán (v.v. 28-IV-1968).

Daucus muricatus (L.) L.

TF93 Carretera de Casares (v.v. 7-V-1970).

ERICALES

ERICACEAE

Erica arbborea L.

TF93 way to Majada Madrid (v.v. 14-II-1974).

UF14 Carretera 339 (v.v. 10-II-1968). 
Erica erigena R. Ross

UF03 Sierra Bermeja (v.v. 3-V-1972).

UF15 Carretera 339 (v.v. 11-IV-1974).

Erica scoparia L.

UF03 Sierra Bermeja (v.v. 3-V-1972).

Erica terminalis Salisb.

UF03 Sierra Bermeja, 12-V-1971, Smythies (Herb. LTR 564).

UF14 Benahavís (v.v. 13-IV-1973).

UF15 Carretera 339 (v.v. 11-IV-1974).

\section{PRIMULALES \\ PRIMULACEAE}

Androsace maxima $\mathrm{L}$.

UF16 Sierra de las Nieves (v.v. 29-V-1975).

Asterolinon linum-stellatum (L.) Duby

UF04 Sierra Bermeja (v.v. 28-III-1973).

Anagallis crassifolia Thore

UF03 Sierra Bermeja (v.v. 3-V-1972).

UF14 Benahavís (v.v. 13-IV-1973).

Coris monspeliensis $\mathrm{L}$.

UF03 Sierra Bermeja (v.v. 9-V-1969).

UF65 Sierra Llana (v.v. 24-V-1971).

VF17 above Canillas de Albaida (v.v. 17-V-1972).

\section{PLUMBAGINALES}

PLUMBAGINACEAE

\section{Armeria colorata Pau}

UF04 Sierra Bermeja, 28-V-1970, Smythies (Herb. LTR 433).

UF14 Carretera 339 (v.v. 4-V-1967). 
Armeria villosa Girard

UF16 Sierra de las Nieves, 29-V-1975, Smythies (Herb. LTR 1084).

UF26 Sierra de las Nieves, 28-V-1974, Smythies (Herb. LTR 952).

UF55 Sierra de Mijas, 26-V-1974, Smythies (Herb. LTR 947).

VF19 Baños de Alhama (Granada), 13-V-1970, Smythies (Herb. LTR 412).

\section{OLEALES}

OLEACEAE

Jasminum fruticans $\mathrm{L}$.

UF06 Ronda (v.v. 6-V-1967).

\section{GENTIANALES}

GENTIANACEAE

Blackstonia perfoliata (L.) Hudson

UF65 Sierra Llana (v.v. 26-V-1974).

\section{RUBIACEAE}

Putoria calabrica (L. fil.) DC.

UF34 Sierra Blanca, 2-XI-1965, Smythies (Herb. E 6).

\section{Sherardia arvensis $\mathrm{L}$.}

UF03 Sierra Bermeja (v.v. 4-IV-1967).

UF04 Sierra Bermeja (v.v. 4-IV-1967).

\section{Asperula hirsuta Desf.}

UF65 Sierra Llana (v.v. 24-V-1971).

Galium boissieranum Ehrend. \& Krendl

UF03 Sierra Bermeja (v.v. 11-VI-1974).

UF14 Benahavís, 28-IV-1973, Smythies (Herb. LTR 780).

UF55 Sierra de Mijas, 26-V-1974, Smythies (Herb. LTR 945). 


\section{Galium mollugo L.}

UF06 Montejaque (v.v. 21-V-1975).

Galium saccharatum All.

UF14 Benahavís (v.v. 28-IV-1973).

\section{Galium spurium L.}

UF26 Torrecilla, 6-VI-1974, Smythies (Herb. LTR 957).

Valantia hispida L.

UF14 Benahavís (v.v. 28-IV-1973).

Rubia peregrina $\mathrm{L}$.

UF02 Carretera 340 (v.v. 20-IV-1967).

\section{TUBIFLORAE}

CONVOLVULACEAE

Cuscuta epithymum (L.) L.

UF65 Sierra Llana (v.v. 24-V-1971).

Convolvulus lanuginosus Desr.

VF08 north of Alcaucín (v.v. 8-VI-1975).

VF17 above Canillas de Albaida (v.v. 17-V-1972).

Convolvulus siculus $\mathrm{L}$.

UF14 Benahavís (v.v. 25-IV-1966).

\section{BORAGINACEAE}

Neatostema apulum (L.) I. M. Johnston

UF69 Carretera 331 (v.v. 28-III-1966).

Buglossoides arvensis (L.) I. M. Johnston

UF06 Ronda (v.v. 11-IV-1974). 
Lithodora fruticosa (L.) Griseb.

UF16 Sierra de las Nieves (v.v. 29-V-1975).

Echium albicans Lag. \& Rodr.

TF93 Sierra Crestellina (v.v. 26-IV-1968).

UF35 Sierra Blanca (v.v. 29-III-1967).

UF37 Sierra de Alcaparaín (v.v. 14-IV-1967).

UF48 Sierra de la Pizarra (v.v. 28-III-1967).

VF17 above Canillas de Albaida (v.v. 6-V-1969).

Echium flavum Desf.

UF04 Sierra Bermeja (v.v. 4-IV-1967).

UF14 Carretera 339, 5-IV-1966, Smythies (Herb. E 63).

UF15 Coto Ragete, 6-VI-1974, Smythies (Herb. LTR 963).

Echium plantagineum L.

UF67 Almogía, 29-III-1966, Smythies (Herb. E 54).

UF98 Carretera 335, 25-III-1966, Smythies (Herb. E 48).

VF07 Carretera 335, 25-III-1966, Smythies (Herb. E 47).

Myosotis minutiflora Boiss. \& Reuter

UF26 Sierra de las Nieves (v.v. 7-V-1967).

Myosotis ramosissima Rochel

UF14 Benahavís (v.v. 22-II-1973).

UF34 Sierra Blanca (v.v. 13-V-1973).

Omphalodes linifolia (L.) Moench

UF14 Benahavís (v.v. 28-IV-1968).

UF26 Sierra de las Nieves (v.v. 7-V-1967).

UF47 Sierra de Aguas (v.v. 18-V-1970).

Cynoglossum cheirifolium L.

UF55 Sierra de Mijas (v.v. 6-III-1972). 


\section{LABIATAE}

Ajuga chamaepitys (L.) Schreber

UF34 Sierra Blanca (v.v. 15-IV-1967).

Ajuga iva (L.) Schreber

UF02 Carretera 340 (v.v. 29-IV-1970).

Teucrium fragile Boiss.

VF08 north of Alcaucín (v.v. 8-VI-1975).

VF17 above Canillas de Albaida, 6-V-1969, Smythies (Herb. LTR 339).

Teucrium haenseleri Boiss.

UF55 Sierra de Mijas, 26-V-1974, Smythies (Herb. LTR 946).

Teucrium polium $\mathrm{L}$.

UF65 Sierra Llana, 24-V-1971, Smythies (Herb. LTR 574).

VF17 way to Cortijo del Daire (v.v. 17-V-1972).

Sideritis incana L.

VF08 north of Alcaucín, 8-VI-1975, Smythies (Herb. LTR'1109).

Sideritis romana $\mathrm{L}$.

VF26 Cueva de Nerja (v.v. 13-IV-1967).

Phlomis herba-venti L.

UF18 Carretera 341 (v.v. 8-V-1967).

Nepeta nepetella L.

UF69 El Torcal (v.v. 9-V-1967).

Cleonia lusitanica L.

UF18 Carretera 314 (v.v. 8-V-1967).

UF65 Sierra Llana (v.v. 24-V-1971).

Micromeria graeca (L.) Bentham. ex Reichenb.

UF14 Benahavís (v.v. 25-IV-1966).

VF17 above Canillas de Albaida, 18-V-1972, Smythies (Herb. LTR 710). 
Thymus baeticus Boiss. ex Lacaita.

UF34 Sierra Blanca (v.v. 13-V-1973).

Thymus granatensis Boiss.

UF26 Sierra de las Nieves (v.v. 28-V-1974).

UF37 Sierra de Alcaparaín, 18-V-1970, Smythies (Herb. LTR 427).

Thymus longiflorus Boiss.

VF08 north of Alcaucín, 8-VI-1975, Smythies (Herb. LTR 1108).

Thymus zygis $\mathrm{L}$.

UG50 Carretera 342, 3-VI-1971, Smythies (Herb. LTR 583).

Rosmarinus officinalis $\mathrm{L}$.

UF26 Sierra de las Nieves (v.v. 26-III-1972).

UF35 Sierra Blanca (v.v. 15-IV-1967).

UF48 Sierra de la Pizarra (v.v. 14-IV-1967).

VF18 south of Jatar (v.v. 14-V-1970).

Lavandula lanata Boiss.

VF08 Sierra Tejeda (v.v. 25-V-1974).

VF17 above Canillas de Albaida (v.v. 6-V-1969).

Lavandula multifida L.

VF17 way to Cortijo del Daire (v.v. 17-V-1972).

Salvia argentea L.

UF18 Carretera 341 (v.v. 8-V-1967).

UF55 Sierra de Mijas (v.v. 25-V-1971).

Salvia candelabrum Boiss.

TF93 Casares (v.v. 31-V-1974).

UF37 Sierra de Alcaparaín, 18-V-1970, Smythies (Herb. LTR 423).

VF17 way to Cortijo del Daire (v.v. 17-V-1972).

Salvia lavandulifolia Vahl

VF08 Sierra Tejeda, 25-V-1974, Smythies (Herb. LTR 941). 
Salvia viridis $\mathrm{L}$.

TF94 Sierra Espartina (v.v. 5-V-1975).

UF35 Sierra Blanca (v.v. 29-III-1967).

UF48 Sierra de la Pizarra, 28-III-1967, Smythies (Herb. E 168).

\section{SCROPHULARIACEAE}

Verbascum laciniatum (Poiret) $\mathrm{O}$. Kuntze

TF94 Genalguacil (v.v. 10-V-1972).

Verbascum rotundifolium Ten.

subsp. haenseleri (Boiss.) Murb.

TF84 Sierra del Hacho, Gaucín, 2-V-1968, Smythies (Herb. E 261).

UF14 Carretera 339 (v.v. 4-V-1967).

UF55 Sierra de Mijas (v.v. 12-IV-1975).

VF17 above Canillas de Albaida, 6-V-1969, Smythies (Herb. LTR 328).

Verbascum virgatum Stokes

UF02 Casares road (v.v. 20-IV-1967).

Scrophularia canina $L$.

UF14 Benahavís (v.v. 22-II-1973).

UF35 Sierra Blanca (v.v. 29-III-1967).

\section{Scrophularia scorodonia L.}

UF03 Sierra Bermeja, 11-VI-1974, Smythies (Herb. LTR 974).

Anarrhinum bellidifolium (L.) Willd.

UF03 Sierra Bermeja (v.v. 6-V-1968).

UF14 Carretera 339, 19-II-1966, Smythies (Herb. E 22).

Antirrhinum graniticum Rothm.

VF19 Baños de Alhama (Granada) 25-III-1966, Smythies (Herb. E 51).

VF19 Baños de Alhama (Granada) 13-V-1970, Smythies (Herb. LTR 413).

Antirrhinum majus $\mathrm{L}$.

UF03 Sierra Bermeja (v.v. 12-V-1971). 
Chaenorhinum rubrifolium (Robill. \& Cast. ex DC.) Fourr.

UF04 Sierra Bermeja, 28-V-1970, Smythies (Herb. LTR 435).

UF34 Sierra Blanca (v.v. 13-V-1973).

UF35 Sierra Blanca (v.v. 8-V-1968).

VF17 above Canillas de Albaida, 6-V-1969, Smythies (Herb. LTR 331).

Linaria aeruginea (Gouan.) Cav.

UF35 Sierra Blanca (v.v. 15-IV-1967).

Linaria amethystea (Lam.) Hoffmanns et Link

UF06 Ronda (v.v. 6-V-1967).

UF34 Sierra Blanca, 29-III-1967, Smythies (Herb. E 169).

UF48 Sierra de la Pizarra, 14-IV-1967, Smythies (Herb. E 181).

Linaria amoi Campo ex Amo

UF98 Ventas de Zafarraya (Granada) 25-III-1966, Smythies (Herb. E 49).

VF08 north of Alcaucín, 8-VI-1975, Smythies (Herb. LTR 1110).

VF17 above Canillas de Albaida (v.v. 17-V-1972).

VF18 south of Jatar (Granada) (v.v. 14-V-1970).

Linaria anticaria Boiss. et Reuter

UF69 El Torcal, 28-III-1966, Smythies (Herb. E 53).

VF19 Baños de Alhama (Granada) 25-III-1966, Smythies (Herb. E 51).

VF19 Baños de Alhama (Granada) 13-V-1970, Smythies (Herb. LTR 411).

Linaria clementei Haenseler ex Boiss.

UF35 Sierra Blanca, 2-XI-1965, Smythies (Herb. E 7).

UF37 Sierra de Alcaparaín (v.v. 14-IV-1967).

UF55 Sierra de Mijas, 26-V-1974, Smythies (Herb. LTR 944).

Linaria hirta (L.) Moench

VF19 Carretera 340 (v.v. 13-V-1970).

Linaria huteri Lange

UF55 Sierra de Mijas, 26-V-1974, Smythies (Herb. LTR 948).

Linaria platycalix Boiss.

UF06 Montejaque (v.v. 21-V-1975). 


\section{Linaria saturejoides Boiss.}

UF14 Benahavís (v.v. 22-II-1973).

VF17 above Canillas de Albaida, 6-V-1969, Smythies (Herb. LTR 330).

\section{Linaria tristis (L.) Miller}

UF03 Sierra Bermeja, 4-IV-1967, Smythies (Herb. E 173).

UF06 Ronda (v.v. 6-V-1967).

UF14 Benahavís (v.v. 22-II-1973).

\section{Linaria verticillata Boiss.}

UF89 Sierra de Camorolos, 24-V-1974, Smythies (Herb. LTR 930).

VF18 south of Jatar (Granada), 14-V-1970, Smythies (Herb. LTR 414).

\section{Linaria viscosa (L.) Dum-Courset}

UF48 Sierra de la Pizarra, 28-III-1967, Smythies (Herb. E 165).

UF69 El Torcal, 23-V-1974, Smythies (Herb. LTR 926).

VF17 above Canillas de Albaida, 6-V-1969, Smythies (Herb. LTR 336).

VF17 above Canillas de Albaida, 18-V-1972, Smythies (Herb. LTR 709).

\section{Digitalis obscura $\mathrm{L}$.}

subsp. laciniata (Lindley) Maire

UF14 Benahavís (v.v. 3-V-1970) Carretera 339 (v.v. 4-V-1967).

VF17 way to Cortijo del Daire (v.v. 17-V-1972).

\section{Erinus alpinus $\mathrm{L}$.}

UF26 Sierra de las Nieves (v.v. 28-V-1974).

\section{Veronica agrestis $\mathrm{L}$.}

UF14 Benahavís (v.v. 22-II-1973).

\section{Veronica cymbalaria Bodard}

TF85 Peñón de Berrueco, 5-V-1971, Smythies (Herb. LTR 560).

TF93 way to Majada Madrid (v.v. 14-II-1974):

Veronica praecox All.

UF16 Sierra de las Nieves (v.v. 29-V-1975). 
Parentucellia latifolia (L.) Caruel

TF97 Carretera 344 (v.v. 4-IV-1969).

Bellardia trixago (L.) All.

UF69 Carretera 331 (v.v. 29-III-1966).

\section{ACANTHACEAE}

Acanthus mollis $\mathrm{L}$.

UF14 Benahavís (v.v. 3-V-1970).

\section{OROBANCHACEAE}

Orobanche calendulae Pomel

TF96 Cueva de la Pileta, 21-V-1974, Smythies (Herb. LTR 918).

UF55 Sierra de Mijas, 26-V-1974, Smythies (Herb. LTR 950).

Orobanche foetida Poiret

UF34 Ojén, 13-V-1973, Smythies (Herb. LTR 801).

Orobanche gracilis $\mathrm{Sm}$.

TF98 El Castor (v.v. 27-V-1975).

Orobanche haenseleri Reuter

UF26 Sierra de las Nieves (v.v. 6-VI-1974).

Orobanche latisquama (F. W. Schultz) Batt.

UF14 Benahavís (v.v. 13-IV-1973).

Orobanche minor $\mathrm{Sm}$.

UF14 Benahavís (v.v. 13-IV-1973).

Orobanche purpurea Jacq.

UF06 Ronda (v.v. 6-V-1967). 


\title{
Orobanche ramosa $\mathrm{L}$.
}

UF14 Benahavís (v.v. 28-IV-1968).

Orobanche rapum-genistae Thuill.

VF17 above Canillas de Albaida (v.v. 6-V-1969).

\section{PLANTAGINALES}

\section{PLANTAGINACEAE}

\section{Plantago albicans L.}

VF17 above Canillas de Albaida, 18-V-1972, Smythies (Herb. LTR 705).

Plantago amplexicaule Cav.

UF65 Torremolinos, 25-IV-1972, Smythies (Herb. LTR 649).

\author{
DIPSACALES
}

CAPRIFOLIACEAE

\section{Viburnum tinus $\mathrm{L}$.}

UF14 Benahavís (v.v. 12-I-1968).

\section{VALERIANACEAE}

\section{Valeriana tuberosa $\mathrm{L}$.}

UF03 Sierra Bermeja (v.v. 28-IV-1973).

UF55 Sierra de Mijas (v.v. 23-IV-1975).

UF89 Sierra de Camorolos, 24-V-1974, Smythies (Herb. LTR 927).

Centranthus calcitrapa (L.) Dufresne

UF37 Sierra de Alcaparaín (v.v. 23-IV-1970).

\section{DIPSACACEAE}

Scabiosa turolensis Pau ex Willk.

UF48 Camino del Rey, 10-V-1974, Smythies (Herb. LTR 907). 


\section{Campanula erinus L.}

UF48 Camino del Rey (v.v. 10-V-1974).

\section{Campanula lusitanica L.}

UF14 Benahavís (v.v. 10-VI-1973).

Trachelium coeruleum L.

UF03 Sierra Bermeja (v.v. 12-V-1971).

UF35 Sierra Blanca (v.v. 2-XI-1965).

UF48 Camino del Rey (v.v. 10-V-1974).

Jasione montana L.

UF14 Benahavís, 10-VI-1973, Smythies (Herb. LTR 836).

UF49 Sierra de Abdalajís, 24-V-1974, Smythies (Herb. LTR 933).

\section{COMPOSITAE}

Filago fuscescens Pomel

UF49 Sierra de Abdalajís, 24-V-1974, Smythies (Herb. LTR 932b).

\section{Filago pyramidata L.}

UF16 Sierra de las Nieves, 6-VI-1974, Smythies (Herb. LTR 962b).

Evax pygmaea (L.) Brot.

UF06 Ronda, 2-IV-1974, Smythies (Herb. LTR 899).

Bombycilaena discolor (Pers.) Lainz

=Micropus bombycinus Lag.

UF49 Sierra de Abdalajís, 24-V-1974, Smythies (Herb. LTR 932a).

Micropus supinus L.

UF16 Sierra de las Nieves, 6-VI-1974, Smythies (Herb. LTR 962). 
Helichrysum serotinum Boiss.

VF17 above Canillas de Albaida, 6-V-1969, Smythies (Herb. LTR 334).

Phagnalon rupestre (L.) DC.

UF65 Sierra Llana (v.v. 24-V-1971).

Phagnalon saxatilis (L.) Cass.

UF65 Sierra Llana (v.v. 24-V-1971).

Pulicaria odora (L.) Reichenb.

UF03 Sierra Bermeja (v.v. 4-IV-1967).

UF04 Sierra Bermeja (v.v. 4-IV-1967).

Cladanthus arabicus (L.) Cass.

UF65 Torremolinos, 25-IV-1972, Smythies (Herb. LTR 648).

UF67 Almogía (v.v. 29-III-1966).

Anacyclus clavatus (Desf.) Pers.

TF96 Montejaque (v.v. 21-V-1975).

Prolongoa pectinata (L.) Boiss.

UF06 Ronda (v.v. 3-IV-1969).

UF15 Carretera 339 (v.v. 11-IV-1974).

UF17 Puerto de El Viento, 4-III-1966, Smythies (Herb. E 36).

Coleostephus myconis (L.) Reichenb. fil.

UF03 Río Padrón (v.v. 7-IV-1972).

Leucanthemum arundanum (Boiss.) Cuatrec.

UF26 Torrecilla, 6-VI-1974, Smythies (Herb. LTR 956).

Leucanthemum paludosum (Poiret) Bonnet \& Barratte

=Hymenostema fontanesii Willk.

UF06 Montejaque, 4-III-1966, Smythies (Herb. E 35).

Doronicum pardalianches L.

UF15 Coto Ragete (v.v. 3-IV-1969). 
Senecio eriopus Willk.

UF04 Sierra Bermeja, 4-IV-1967, Smythies (Herb. E 170).

UF65 Sierra Llana (v.v. 26-V-1974).

Senecio gallicus Chaix

UF35 Sierra Blanca, 13-III-1973, Smythies (Herb. LTR 752).

Senecio minutus (Cav.) DC.

TF93 Sierra Crestellina (v.v. 26-IV-1968).

UF14 Benahavís, 28-III-1973, Smythies (Herb. LTR 755).

UF15 Carretera 339 (v.v. 4-V-1967).

UF34 Sierra Blanca, 15-IV-1967, Smythies (Herb. E 180).

Senecio petraeus Boiss. \& Reuter

TF93 Sierra Crestellina (v.v. 12-V-1975).

\section{Calendula arvensis $\mathrm{L}$.}

VF17 above Canillas de Albaida, 18-V-1972, Smythies (Herb. LTR 703).

Calendula suffruticosa Vahl subsp. suffruticosa

UF14 Benahavís (v.v. 3-V-1970).

UF98 Ventas de Zafarraya (Granada), 13-V-1970, Smythies (Herb. LTR 409).

Staehelina baetica DC.

UF04 Sierra Bermeja, 12-V-1971, Smythies (Herb. LTR 561).

Jurinea humilis (Desf.) DC.

VF08 Sierra Tejeda, 25-V-1974, Smythies (Herb. LTR 936).

Jurinea pinnata (Lag.) DC.

UF26 Sierra de las Nieves, 28-V-1974, Smythies (Herb. LTR 953).

UF37 Sierra de Alcaparaín (v.v. 18-V-1970).

Carduus granatensis Willk.

UF14 Carretera 339 (v.v. 4-V-1967). 


\section{Carduus pycnocephalus $\mathrm{L}$.}

UF02 Carretera 340 (v.v. 20-IV-1967).

\section{Carduus tenuiflorus Curtis}

UF14 Benahavís, 28-IV-1973, Smythies (Herb. LTR 781).

\section{Notobasis syriaca Cass.}

UF02 Carretera 340 (v.v. 20-IV-1967).

Ptilostemon hispanicus (Lam.) W. Greuter

=Chamaepeuce hispanica (Lam.) DC.

UF06 Ronda (v.v. 6-V-1967).

UF37 Sierra de Alcaparaín (v.v. 18-V-1970).

Tyrimnus leucographus (L.) Cass.

TF93 Sierra Crestellina (v.v. 12-V-1975).

UF35 Sierra Blanca (v.v. 15-IV-1967).

Cynara humilis L.

UF02 Carretera 340 (v.v. 20-IV-1967).

Serratula baetica Boiss.

UF03 Sierra Bermeja, 11-VI-1974, Smythies (Herb. LTR 968).

UF47 Sierra de Aguas, 18-V-1970, Smythies (Herb. LTR 426).

Serratula flavescens (L.) Poiret

UF65 Sierra Llana, 24-V-1971, Smythies (Herb. LTR 569).

UF65 Sierra Llana, 26-V-1974, Smythies (Herb. LTR 943).

Serratula pinnatifida (Cav.) Poiret

TF94 Sierra Espartina (v.v. 5-V-1975).

UF37 Sierra de Alcaparaín (v.v. 18-V-1970).

Leuzaea conifera (L.) DC.

UF35 Sierra Blanca (v.v. 15-IV-1967).

VF08 Sierra Tejeda (v.v. 25-V-1974).

Cyanopsis muricata (L.) Dostál

=Amberboa muricata (L.) DC.

UF67 Almogía, 29-III-1966, Smythies (Herb. E 59). 
Centaurea bombycina Boiss. ex DC.

VF17 above Canillas de Albaida, 18-V-1972, Smythies (Herb. LTR 706).

Centaurea carratracensis Lange

UF47 Sierra de Aguas, 18-V-1970, Smythies (Herb. LTR 424).

\section{Centaurea eriophora L.}

UG50 Carretera 342, 3-VI-1971, Smythies (Herb. LTR 582).

Centaurea haenseleri (Boiss.) Boiss.

UF04 Sierra Bermeja, 28-V-1970, Smythies (Herb. LTR 437).

\section{Centaurea melitensis L.}

UF47 Sierra de Aguas, 18-V-1970, Smythies (Herb. LTR 425).

UF65 Sierra Llana, 24-V-1971, Smythies (Herb. LTR 572).

Centaurea prolongi Boiss. ex DC.

UF65 Sierra Llana, 24-V-1971, Smythies (Herb. LTR 568).

\section{Centaurea seridis $\mathrm{L}$.}

VF17 above Canillas de Albaida, 18-V-1972, Smythies (Herb. LTR 702).

Centaurea triumfetti All.

subsp. triumfetti

$=$ Centaurea variegata Lam.

UF26 Torrecilla (v.v. 6-VI-1974).

VF08 Sierra Tejeda (v.v. 25-V-1974).

Crupina crupinastrum (Moris) Vis.

UF06 Montejaque (v.v. 6-V-1967).

UF65 Sierra Llana, 24-V-1971, Smythies (Herb. LTR 571).

VF19 Baños de Alhama (Granada) (v.v. 13-V-1970).

Carduncellus caeruleus (L.) C. Presl

UF02 Carretera 340 (v.v. 20-IV-1967).

Scolymus maculatus $\mathrm{L}$.

UF17 Carretera 341, 3-VI-1971, Smythies (Herb. LTR 581). 
Scorzonera baetica (Boiss.) Boiss.

UF03 Sierra Bermeja (v.v. 6-V-1968).

UF04 Sierra Bermeja (v.v. 6-V-1968).

UF65 Sierra Llana, 24-V-1971, Smythies (Herb. LTR 573).

Scorzonera crispatula (Boiss.) Boiss.

UF35 Sierra Blanca (v.v. 13-V-1973).

\title{
Scorzonera graminifolia L.
}

UF65 Sierra Llana, 24-V-1971, Smythies (Herb. LTR 570).

Lactuca viminea (L.) J. \& C. Presl subsp. ramosissima (All.) Bonnier

UF03 Sierra Bermeja, 3-VI-1975, Smythies (Herb. LTR 1090).

Andryala ragusina $\mathrm{L}$.

UF14 Benahavís, 27-XI-1969, Smythies (Herb. LTR 389).

\author{
SPERMATOPHYTA \\ ANGIOSPERMAE \\ MONOCOTYLEDONES
}

LILIIFLORAE

LILIACEAE

Aphyllanthes monspeliensis $\mathrm{L}$.

TF93 Sierra Crestellina (v.v. 26-IV-1968).

UF03 Sierra Bermeja (v.v. 6-V-1968).

Asphodelus cerasiferus Gay

UF03 Sierra Bermeja, 12-V-1971, Smythies (Herb. LTR 563).

UF65 Sierra Llana, 24-V-1971, Smythies (Herb. LTR 575).

\section{Asphodelus fistulosus L.}

UF43 Artola, 16-II-1970, Smythies (Herb. LTR 393).

Colchicum atticum Spruner ex Tommas

UF16 Ronda, 19-III-1970, Smythies (Herb. LTR 633). 
Anthericum baeticum Boiss.

UF03 Sierra Bermeja (v.v. 28-IV-1973).

UF04 Sierra Bermeja, 28-V-1970, Smythies (Herb. LTR 436).

UF14 Benahavís (v.v. 28-IV-1973).

Gagea nevadensis Boiss.

UF04 Sierra Bermeja (v.v. 4-IV-1967).

UF15 Carretera 339, 19-II-1966, Smythies (Herb. E 23).

Allium neapolitanum Cyr.

UF48 Camino del Rey (v.v. 10-V-1974).

Fritillaria hispanica Boiss. \& Reuter

UF04 Sierra Bermeja (v.v. 4-IV-1967).

Tulipa australis $\mathrm{L}$.

UF04 Sierra Bermeja (v.v. 4-IV-1967).

VF08 Sierra Tejeda (v.v. 25-V-1974).

Tulipa praecox Ten.

UF06 Ronda, 11-VI-1974, Smythies (Herb. LTR 904).

Endymion hispanicum (Miller) Chouard

TF93 Sierra Crestellina (v.v. 26-IV-1968).

UF67 El Torcal (v.v. 28-III-1966).

Ornithogalum reverchoni Lange

UF06 Montejaque, 4-III-1966, Smythies (Herb. E 56).

\section{AMARYLLIDACEAE}

Narcissus cantabricus DC.

UF14 Benahavís, 27-I-1966, Smythies (Herb. E 13).

UF15 Carretera 339 (v.v. 19-II-1966).

Narcissus gaditanus Boiss. \& Reuter

UF55 Sierra de Mijas (v.v. 13-III-1973). 


\section{Narcissus jonquilla L.}

TF93 Carretera 339 (v.v. 19-III-1972).

Narcissus pseudonarcissus $\mathrm{L}$.

subsp. major (Curtis) Baker

$=N$. hispanicus Gou

UF15 Coto Ragete, 19-III-1972, Smythies (Herb. LTR 631).

Narcissus requienii Roemer

UF15 Carretera 339 (v.v. 4-III-1966).

UF16 Carretera 339, 19-III-1972, Smythies (Herb. LTR 632).

UF26 Sierra de las Nieves (v.v. 7-V-1967).

UF27 El Burgo-Ardales road, 27-III-1972, Smythies (Herb. LTR 635).

UF34 Sierra Blanca (v.v. 28-III-1967).

UF35 Sierra Blanca (v.v. 28-III-1967).

\section{IRIDACEAE}

Crocus nevadensis Amo \& Campo

UF04 Sierra Bermeja, 13-I-1967, Smythies (Herb. E 142).

UF04 Sierra Bermeja, 26-I-1968, Smythies (Herb. E 239).

Romulea bulbocodium Kunze

UF04 Sierra Bermeja (v.v. 4-IV-1967).

UF05 Sierra Bermeja (v.v. 7-IV-1972).

Romulea uliginosa Kunze

UF26 Sierra de las Nieves, 26-III-1972, Smythies (Herb. LTR 638).

Iris filifolia Boiss.

UF03 Sierra Bermeja (v.v. 9-V-1969).

UF35 Sierra Blanca (v.v. 15-IV-1967).

UF47 Sierra de Aguas (v.v. 18-V-1970).

UF55 Sierra de Mijas (v.v. 25-V-1971).

Iris lutescens Lam.

subsp. subbiflora

UF69 El Torcal (v.v. 2-V-1965). 
Iris planifolia (Miller) Fiori \& Paol.

TF95 Atajate, 25-XII-1967, (Herb. E 241).

TF97 Carretera 339/344, (v.v. 12-III-1972).

UF06 Ronda (v.v. 12-III-1972).

Iris xiphium $L$.

UF03 Sierra Bermeja (v.v. 4-IV-1967).

VF08 north of Alcaucín (v.v. 9-VI-1975).

JUNCACEAE

Juncus acutus $\mathrm{L}$.

UF03 Río Padrón (v.v. 19-II-1973).

SPADICIFLORAE

PALMAE

Chamaerops humilis $\mathrm{L}$.

UF24 La Concha (v.v. 24-II-1973).

\section{GLUMIFLORAE \\ GRAMINEAE}

Echinaria capitata Desf.

UF16 Sierra de las Nieves (v.v. 29-V-1975).

Arundo plinii Turr.

UF14 Benahavís (v.v. 22-II-1973).

Melica minuta L.

UF04 Sierra Bermeja, 3-V-1972, Smythies (Herb. LTR 677).

UF14 Benahavís, 28-III-1973, Smythies (Herb. LTR 756).

Poa bulbosa $\mathrm{L}$.

UF04 Sierra Bermeja, 3-V-1972, Smythies (Herb. LTR 675).

UF04 Sierra Bermeja, 3-V-1972, Smythies (Herb. LTR 678. [var. vivipara] 
Poa ligulata Boiss.

UF16 Sierra de las Nieves, 29-V-1975, Smythies (Herb. LTR 1089).

Lepturus filiformis Tim.

UF04 Sierra Bermeja, 20-V-1974, Smythies (Herb. LTR 911).

Arrhenaterum elatius $\mathrm{M}$. K.

UF04 Sierra Bermeja, 3-V-1972, Smythies (Herb. LTR 676).

Stipa capensis Thunb.

$=S$. tortilis Desf.

UF24 Istán, 3-V-1975, Smythies (Herb. LTR 1059).

Nardurus salzmannii Boiss.

UF14 Benahavís, 10-VI-1973, Smythies (Herb. LTR 837).

\section{CYPERALES}

CYPERACEAE

Carex halleriana Ass.

UF24 La Concha (v.v. 24-II-1973).

Carex hispida W.

UF14 Benahavís, 13-IV-1973, Smythies (Herb. LTR 769).

Holoschoenus vulgaris Link

=Scirpus holoschoenus L.

UF03 Sierra Bermeja (v.v. 20-V-1974).

\section{ORCHIDALES}

ORCHIDACEAE

Ophrys apifera Huds.

TF93 Sierra Crestellina (v.v. 12-V-1975).

UF14 Benahavís (v.v. 25-IV-1966).

UF37 Sierra de Alcaparaín (v.v. 14-IV-1967).

VF08 north of Alcaucín (v.v. 8-VI-1975). 


\section{Ophrys atlantica Murby}

UF37 Sierra de Alcaparaín (v.v. 14-IV-1967).

\section{Ophrys fusca L.}

UF37 Sierra de Alcaparaín (v.v. 14-IV-1967).

UF55 Sierra de Mijas (v.v. 6-III-1972).

UF69 Carretera 331 (v.v. 29-III-1966).

VF17 way to Cortijo del Daire (v.v. 17-V-1972).

Ophrys lutea Cav.

TF93 Sierra Crestellina (v.v. 12-V-1975).

UF37 Sierra de Alcaparaín (v.v. 14-IV-1967).

UF69 Carretera 331 (v.v. 29-III-1966).

VF17 way to Cortijo del Daire (v.v. 17-V-1972).

Ophrys scolopax Cav.

UF14 Benahavís (v.v. 25-IV-1966).

UF37 Sierra de Alcaparaín (v.v. 14-IV-1967).

VF08 Sierra Tejeda (v.v. 25-V-1974).

Ophrys speculum Link

UF37 Sierra de Alcaparaín (v.v. 14-IV-1967).

Ophrys tenthredinifera Willd.

TF93 Sierra Crestellina (v.v. 12-V-1975).

UF14 Benahavís (v.v. 25-IV-1966).

Orchis italica Poiret

TF93 Sierra Crestellina (v.v. 26-IV-1968).

UF15 Carretera 339 (v.v. 4-V-1967).

UF35 Sierra Blanca (v.v. 15-IV-1967).

Orchis laxiflora Lam.

UF14 Carretera 339 (v.v. 5-IV-1966).

Orchis lactea Poiret

UF15 Carretera 339 (v.v. 4-III-1966).

UF55 Sierra de Mijas (v.v. 6-III-1972). 
Orchis mascula L.

TF93 Sierra Crestellina (v.v. 26-IV-1968).

UF04 Sierra Bermeja (v.v. 6-V-1968).

UF34 Sierra Blanca (v.v. 29-III-1967).

UF35 Sierra Blanca (v.v. 15-IV-1967).

UF37 Sierra de Alcaparaín (v.v. 14-IV-1967).

UF55 Sierra de Mijas (v.v. 23-IV-1975).

UF69 Carretera 331 (v.v. 29-III-1966).

Orchis morio L.

UF16 Carretera 339 (v.v. 4-V-1967).

Orchis pallens $\mathrm{L}$.

UF04 Sierra Bermeja (v.v. 6-V-1968).

UF34 Sierra Blanca (v.v. 15-IV-1967).

Orchis papilionacea L.

UF37 (v.v. 6-III-1966).

UF69 Carretera 331 (v.v. 29-III-1966).

Orchis saccata Ten.

UF55 Sierra de Mijas (v.v. 6-III-1972).

UF69 Carretera 331 (v.v. 29-III-1966).

Aceras anthropophorum R. Br.

TF93 Sierra Crestellina (v.v. 26-IV-1968).

UF35 Sierra Blanca (v.v. 15-IV-1967).

UF37 Sierra de Alcaparaín (v.v. 14-IV-1967)

UF55 Sierra de Mijas (v.v. 23-IV-1975).

Himantoglossum hircinum (L.) Spreng.

UF55 Sierra de Mijas (v.v. 25-V-1971).

Cephalanthera Iongifolia (L.) Fritsch

UF34 Sierra Blanca (v.v. 15-IV-1967). 
Limodorum trabuttianum Batt.

UF03 Sierra Bermeja (v.v. 12-V-1971).

UF05 Alpandeire (v.v. 30-V-1975).

Neotinea intacta (Link) Rchb.

UF35 Sierra Blanca (v.v. 15-IV-1967).

UF37 Sierra de Alcaparaín (v.v. 14-IV-1967).

UF48 Sierra de la Pizarra (v.v. 28-III-1967).

UF55 Sierra de Mijas (v.v. 23-IV-1975).

(Recibido el 10 de octubre de 1976) 